\title{
SERÁ QUE A POPULAÇÃO CONSOME A QUANTIDADE DE PEIXE RECOMENDADA PELA FAO?
}

\author{
Karoline Fontana Simon ${ }^{1}$ \\ Áline Mayara Ferreira ${ }^{2}$ \\ Márcia Fernandes Nishiyama ${ }^{3}$ \\ Everton Artuso ${ }^{4}$
}

\section{Resumo}

Nas últimas décadas a aquicultura recebeu grande atenção, e ainda vem recebendo, devido, em grande parte, pelo consumo de peixes estarem relacionados a benefícios à saúde. Objetivou-se verificar o perfil socioeconômico e identificar o consumo de pescados de indivíduos residentes no município de Seara, localizado no Oeste de Santa Catarina, bem como a quantidade consumida e os motivos, pelos quais consomem mais peixes. A pesquisa foi de natureza qualiquantitativa, descritiva, e de temporalidade transversal. Foram entrevistados 376 indivíduos, utilizando-se de um formulário semi-estruturado composto por 30 questões objetivas e subjetivas. Esta pesquisa foi aprovada pelo Comitê de Ética e Pesquisa da Universidade Federal da Fronteira Sul (UFFS) (CAAE: 1.001.5 - 556). Os resultados encontrados demonstraram que a maior renda e escolaridade influenciam no maior consumo de peixes, pois, conforme aumentou o grau de escolaridade e a renda, também aumentou a quantidade de pescados consumidos pelos entrevistados. Percebeu-se ainda, que grande parte $(90,7 \%)$ dos participantes consumiam peixes, porém, a quantidade consumida encontrava-se abaixo do recomendado. Dentre os motivos citados pelos participantes, que fariam aumentar, seu consumo de pescados estariam: os preços mais acessíveis e incentivos.

Palavras-chave: consumo alimentar; pescado; recomendação nutricional; aquicultura.

\section{Introdução}

A aquicultura apesar de ser uma técnica milenar e de subsistência, ganhou novo impulso somente nas últimas décadas e está se apresentando como uma atividade em franca expansão no Brasil, tendo em vista a demanda mundial a estes alimentos (MINISTÉRIO DA PESCA E AQUICULTURA, 2011).

\footnotetext{
${ }^{1}$ Graduada em Bacharel em Nutrição na Universidade Federal da Fronteira Sul - UFFS. Mestranda em Ciência e Tecnologia de Alimentos na Universidade do Estado de Santa Catarina - UDESC.

${ }^{2}$ Graduada em Bacharel em Nutrição na Universidade Federal da Fronteira Sul - UFFS. Mestranda em Desenvolvimento Regional na Universidade Tecnológica Federal do Paraná - UTFPR.

${ }^{3}$ Doutora. Docente em Universidade Federal da Fronteira Sul - UFFS.

${ }^{4}$ Mestre. Docente na Universidade Federal da Fronteira Sul - UFFS.
} 
De acordo com o Regulamento de Inspeção Industrial e Sanitária dos Produtos de Origem Animal - RIISPOA, a partir do Decreto $n^{0} 30.691$, de 1952, ao termo pescado se definem os peixes, crustáceos, moluscos, anfíbios, quelônios e mamíferos de água doce ou salgada, utilizados na alimentação humana (BRASIL, 1952).

Devido ao valor nutricional do pescado e a elaboração de pesquisas que o associem com melhorias à saúde, ultimamente tem ocorrido um acréscimo de interesse por esse alimento (BURGUER, 2008). Segundo Prado e Capuano (2006) a sua ingestão, se relaciona intimamente com alimentação saudável, visto que possui nível protéico alto, baixa taxa de gordura e presença de ácidos graxos insaturados.

De acordo com a Organização das Nações Unidas para Alimentação e Agricultura (FAO), no Brasil entre 2000 e 2009 o consumo de peixe per capita aumentou cerca de $30 \%$, enquanto o de carne bovina cresceu 10\% (PORTAL BRASIL, 2013). Esse aumento deve-se ao maior poder aquisitivo das famílias (BRASIL, 2013).

De acordo com dados do Ministério da Pesca e Aquicultura de 2013, o brasileiro consumia cerca de $9 \mathrm{~kg}$ de pescado por ano, inferior ao que a Organização das Nações Unidas para Alimentação e Agricultura - FAO recomenda, que é de $12 \mathrm{~kg}$ por pessoa/ano (BRASIL, 2013).

Segundo dados do Sebrae (2010) no estado de Santa Catarina, a pesca desempenha importante papel na economia, sendo considerado o maior estado produtor de pescado e crustáceos do país. Com cerca de 500 quilômetros de litoral, contribui fortemente para que a atividade pesqueira e produtiva do estado seja bastante diversificada, em diversas espécies de peixes, crustáceos e moluscos, capturados por diferentes tipos de embarcações e métodos de pesca.

O ministério da Pesca e Aquicultura (2011) cita o litoral do Espírito Santo; Rio de Janeiro; Paraná e Rio Grande do Sul, destacando a diversidade de ambientes costeiros e inúmeras áreas de criadouros naturais destes estados, indicando que estes ambientes são favoráveis a atividade pesqueira para comunidades inseridas nesta região, e também fonte de subsistência.

Além da importância econômica para algumas regiões do país, destaca-se a importância nutricional do seu consumo para a população em geral, visto que segundo Sartori e Amancio 
(2012) os peixes contêm, uma variedade de vitaminas e minerais: A e D; cálcio, fósforo, ferro, cobre, selênio e, os peixes de água salgada, inclui-se o iodo. Dentre os possíveis benefícios citados pelos autores acima, estão a redução do risco de Acidente Vascular Cerebral (AVC); depressão; Alzheimer e redução de taxa de mortalidade por doenças cardiovasculares.

Sendo assim, os benefícios nutricionais e o seu baixo consumo reforçam a validade de investimentos e incentivos por meio de políticas públicas para o aumento da produção e consumo deste alimento no país (SARTORI; AMANCIO, 2012).

A presente pesquisa justifica-se pela importância do consumo deste alimento à saúde humana, e também no intuito de auxiliar para o aumento de consumo de pescados, na qual segundo os dados aqui apresentados, demonstram a necessidade de estudos e ações nesta temática a nível local, a fim de identificar se a população consome a quantidade recomendada pela Organização das Nações Unidas para Alimentação e Agricultura - FAO, visto seus benefícios, e assim, subsidiar ações educativas, informativas, que auxiliem a consolidação da temática nas diversas regiões do país, frente a sua diversidade territorial, produtiva, econômica e social.

Objetivou-se com a seguinte pesquisa verificar o perfil socioeconômico e identificar o consumo de pescados entre os indivíduos residentes do município de Seara, localizado no Oeste de Santa Catarina, bem como a quantidade consumida e os motivos que, pelos quais consomem mais peixes.

\section{Metodologia}

Pesquisa de natureza quali-quantitativa, descritiva, as fontes de dados e a coleta de campo, e de temporalidade transversal. Realizada entre março a outubro de 2014, no município de Seara, localizado no Oeste do estado de Santa Catarina, onde foram entrevistadas $n=376$ pessoas, a partir do cálculo amostral, considerando o total da população de 16,936 habitantes, segundo dados do Instituto Brasileiro de Geografia e Estatística (IBGE, 2010).

Os entrevistados foram selecionados a partir dos seguintes pré-requisitos: ter maior ou igual a 18 anos e ser residente do município em questão. As entrevistas foram realizadas nas residências das pessoas que assentiram, por meio do termo de consentimento livre e Esclarecido (TCLE). 
Após aprovação do comitê de ética, foi realizado teste-piloto para ajustar o formulário, a fim de atender o objetivo principal da presente pesquisa. O questionário foi estruturado, visando identificar: características socioeconômicas do entrevistado; quantidade de pescado consumido; identificação dos fatores limitantes e/ou facilitadores do consumo de peixes.

Para quantificação do consumo de peixe utilizou-se uma réplica deste alimento, de silicone para exemplificar o tamanho da porção consumida (100 g). E a partir da determinação do consumo per capta e de ingestão (diária, semanal, mensal e anual), foi estimado o consumo anual em quilos e realizada a comparação com a recomendação da FAO.

As entrevistas foram realizadas por um único entrevistador, sendo este a pesquisadora principal, a fim de evitar erros inespecíficos e para manter um padrão individual.

Os resultados obtidos através das variáveis categóricas e qualitativas foram expressos em tabelas e gráficos para interpretação dos dados, sendo utilizado o software SPSS $^{\circledR}$ para realização dos cálculos estatísticos.

Esta pesquisa foi submetida e aprovada pelo Comitê de Ética e Pesquisa da Universidade Federal da Fronteira Sul (UFFS), sob parecer número: 1.001.556. Os entrevistaram receberam o termo de consentimento livre e esclarecido (TCLE) de em que constava, dentre outras, as seguintes: informações detalhadas sobre a pesquisa; a possibilidade de desistir a qualquer momento de participar da pesquisa, sem qualquer prejuízo; as garantias de que sua privacidade seria respeitada; as garantias da confidencialidade das informações pessoais como preconiza a Resolução do Conselho Nacional de Saúde nº CNS no 466 (BRASIL, 2012).

\section{Resultados e Discussão}

Os resultados demonstram que: $59,6 \%$ dos entrevistados pertenciam ao gênero feminino e 40,4\% ao gênero masculino. A idade dos participantes variou entre a mínima de 18 anos e a máxima 86 anos, com média de 36,4 anos.

Em relação à escolaridade dos entrevistados conforme gráfico 1, obteve-se a seguinte distribuição relativa: 9,3\% para "ensino fundamental incompleto"; 10,9\% para "ensino fundamental completo"; 8,8\% para "ensino médio incompleto"; 34,0\% para "ensino médio completo"; $15,4 \%$ para "ensino superior incompleto"; $21,5 \%$ para "ensino superior completo". 


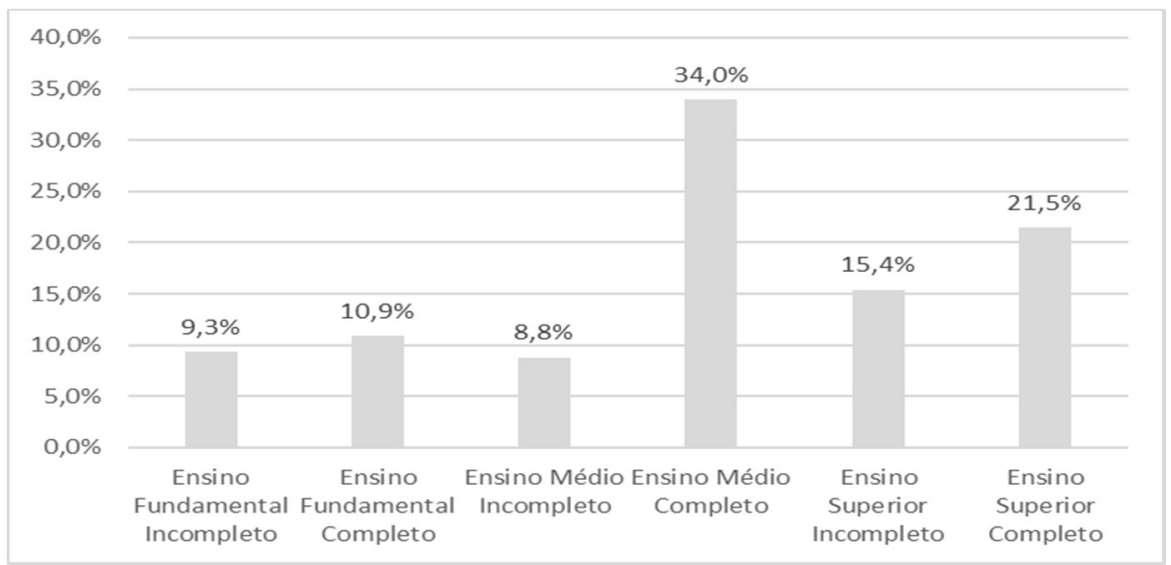

Gráfico 1 - Proporção (\%) da escolaridade dos entrevistados, Seara, SC, 2015.

Fonte: Dados compilados pelos autores, 2014.

A partir da análise estatística, observou-se uma interferência positiva entre duas variáveis: frequência do consumo de peixe e escolaridade das pessoas entrevistadas. Demonstrando que quanto maior era a escolaridade, maior era o consumo de peixe. Diferente dos achados de Vasconcellos (2010), que quando testou a linearidade relacionada ao consumo de peixe, não encontrou associação significativa entre a variável escolaridade e consumo, indicando que o consumo de peixes não aumentava de forma linear à medida que aumentava a escolaridade.

Sobre a renda dos entrevistados, exposto do gráfico 2, observou-se que a grande maioria dos entrevistados possuíam uma renda entre 1 a 3 salários (46\%); os demais: 1,6\%, apresentavam renda menor que 1 salário mínimo; 4 a 6 salários (39,9\%) e mais que 6 salários mínimos eram $12,5 \%$ dos entrevistados.

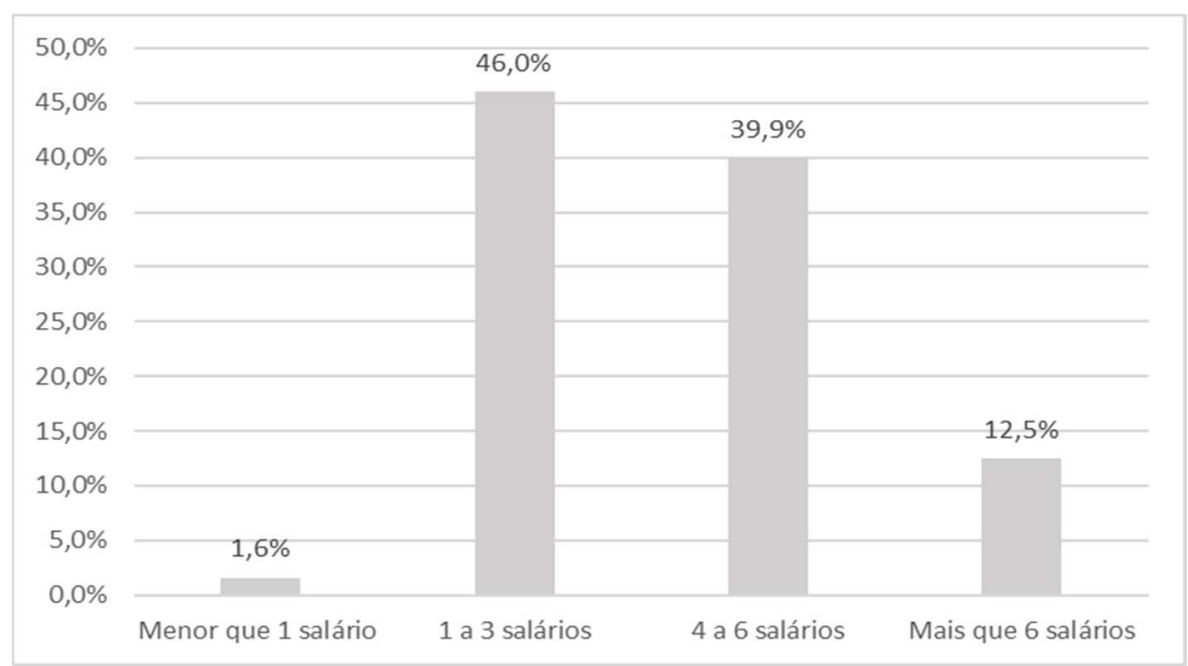

Gráfico 2 - Proporção (\%) da renda bruta mensal familiar dos entrevistados, Seara, SC, 2015.

Fonte: Dados compilados pelos autores, 2014. 
Quando se investigou se havia interferia da renda no consumo de peixes, notou-se que estatisticamente houve uma interferência positiva entre as duas variáveis; ou seja, quanto maior era a renda, maior também era o consumo de peixe.

Vasconcellos (2010) não notou associação significativa entre as variáveis consumo e extratos de renda. No entanto, quando testou a linearidade, observou que a porcentagem de consumo de peixe aumenta quando aumentam os extratos de renda.

Sobre o consumo de peixes, $90,7 \%$ dos entrevistados citaram gostar de consumi-los e $9,3 \%$ negaram o consumo, sendo alguns motivos o fato de não gostar, ter medo de espinhas ou não possuir o hábito. A frequência de consumo maior (35,6\%) foi de 1 vez ao mês; seguida por 1 vez por semana(18,9\%); 2 vezes por mês(17,6\%); 1 a 6 vezes por ano $(9 \%)$ e apenas $9,6 \%$ dos participantes relataram consumir 1 e 2 ou mais vezes por semana como demonstra o Gráfico 3.

Gráfico 3 -Proporção(\%) da frequência do consumo de pescados pelos entrevistados, Seara, SC, 2015.

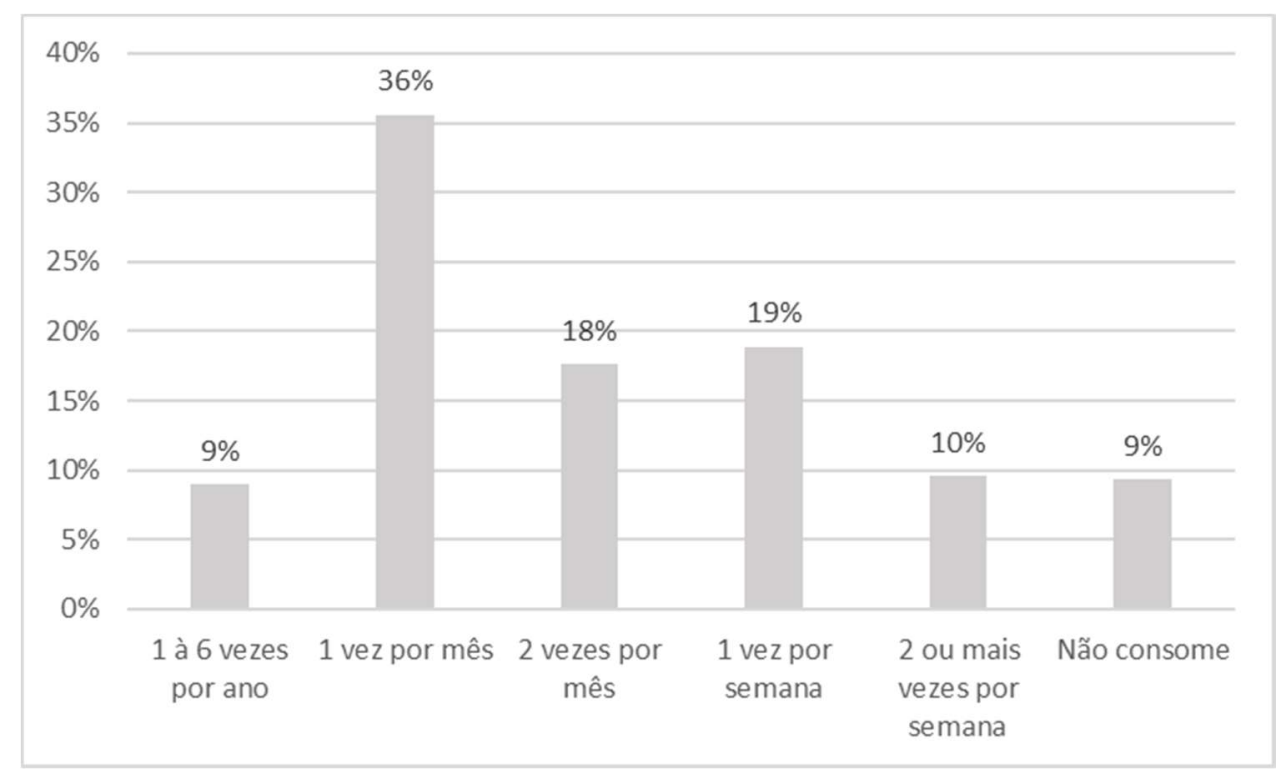

Gráfico 3 -Proporção(\%) da frequência do consumo de pescados pelos entrevistados, Seara, SC, 2015. Fonte: Dados compilados pelos autores, 2014.

Minozzo et al. (2006) realizaram uma pesquisa sobre a frequência do consumo de peixes, e encontrou uma prevalência de $67,42 \%$ dos participantes o hábito de consumir pescados em Toledo, em Curitiba 57,21\% e em São Paulo 83,85\%. Macedo et al. (2014) observaram que a frequência do consumo de peixes que foi mais significativa era de 2 vezes semanais $(21,87 \%)$, seguida de duas vezes por mês $(20,83 \%)$ e $15,63 \%$ declararam consumir 
peixe uma vez por semana. Ainda na mesma pesquisa, 15,63\% relataram consumir peixe três vezes por mês, $12,5 \%$ três vezes por semana, $8,33 \%$ consomem uma vez por mês, $2,08 \%$ uma vez ao ano, 1,04\% quatro vezes por semana, 1,04\% comem peixe cinco vezes por semana e 1,04\% diariamente. Minozzo et al. (2006) também descrevem que 49,69\% dos paulistanos consomem pescado mais que 3 vezes por mês; dados estes, menos representativos em Toledo e Curitiba com 30,81\% e 28,86\%, respectivamente. O Guia Alimentar para a População Brasileira, preconiza o consumo de peixe fresco pelo menos duas vezes por semana (BRASIL, 2008; FAO, 2009).

A análise do consumo per capita permitiu inferir que, na pesquisa atual o valor encontrado foi de $6,63 \mathrm{~kg} / \mathrm{hab} / \mathrm{ano}$, com um desvio padrão de $8,75 \mathrm{~kg}$. Pereira et al. (2010) identificaram consumo per capita de aproximadamente $4,59 \mathrm{~kg} / \mathrm{hab} / \mathrm{ano}$ e que esse valor é variável quando se avalia os extratos por regiões do país; condições geográficas; clima; aspectos socioeconômicos e cultura local.

No entanto, evidencia-se que o consumo brasileiro de pescados está aumentando constantemente, visto que no ano de 2003, o consumo per capita do brasileiro estava situado em 6,5 kg/ano (FAO, 2009; MPA, 2004).

$\mathrm{Na}$ China, o consumo nacional de pescado e produtos da pesca per capita, cresceu de menos de $5 \mathrm{~kg}$ em 1970 para 25,8 kg atualmente, e por conta disso, é responsável pelo grande crescimento da produção (NOMURA, 2010).

Em relação ao motivo pelo qual os participantes da presente pesquisa, aumentariam seu consumo de peixe, obteve-se as seguintes respostas: $31,9 \%$ dos entrevistados citaram que o preço elevado prejudica a compra e consequentemente seu consumo; 19,7\% que deveriam mudar seus hábitos alimentares, no intuito de tornarem mais saudáveis; 19,4\% em razão dos benefícios voltados à saúde; $17,6 \%$ demonstraram que há necessidade de maior incentivo por parte da mídia e órgãos de saúde e 11,4\% não responderam como demonstrado no gráfíco 4.

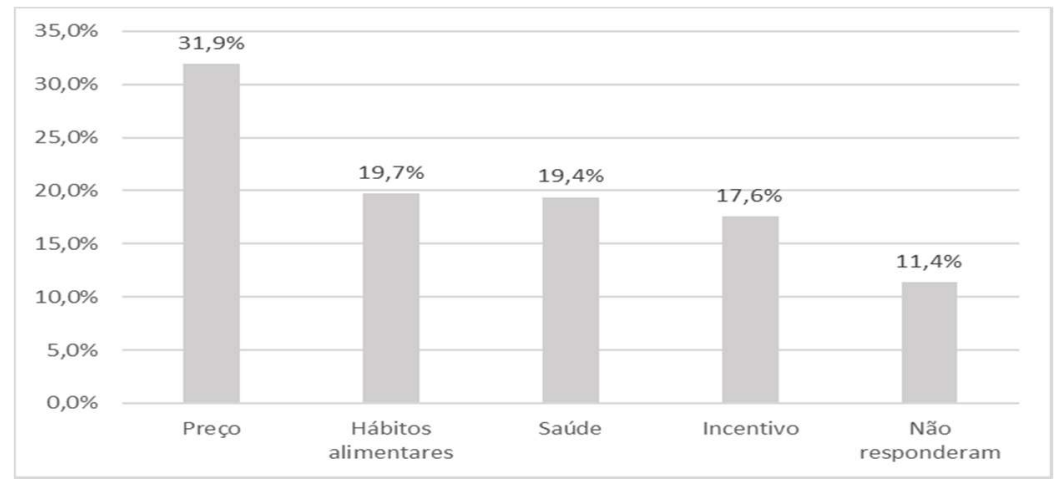

Gráfico 4 -Proporção (\%) dos motivos que fariam os entrevistados consumir mais peixes, Seara, SC, 2015.

Fonte: Dados compilados pelos autores, 2014. 
Barbosa et al. (2007) constataram ao estudar as características comportamentais dos consumidores de pescado no mercado de Belém, que 75,6\% dos entrevistados consideravam que suas decisões de compra eram influenciadas principalmente pelo preço do pescado. Flores et al. (2014) encontraram resultados semelhantes, citando que as principais dificuldades do mercado é preço elevado, e falta da espécie desejada. Além disso, a falta de qualidade, dificuldade no preparo, também foram os motivos citados pelos participantes, sobre o fato de não consumir peixe. Minazzo et al., (2006) corroboram dizendo que dentre as razões que levam a barreira para o consumo de pescado, se relaciona ao preço elevado, que influencia de maneira negativa o seu consumo.

Na presente pesquisa, sobre os motivos que fariam o participantes consumir mais peixe, a variável preço obteve a maior prevalência $(48,4 \%)$, e por conseguinte o acesso $(18,1 \%)$; incentivos e informação nutricional (20,2\%); hábitos alimentares $(4,5 \%)$ e benefícios para à saúde $(7,7 \%)$ e não responderam $(1,1 \%)$, demonstrado no gráfico 5 . Em pesquisa muito semelhante, Vasconcellos (2010) relatou que 54,36\% dos entrevistados, também relataram que um dos motivos que faria a população consumir mais peixes seria a redução do preço.

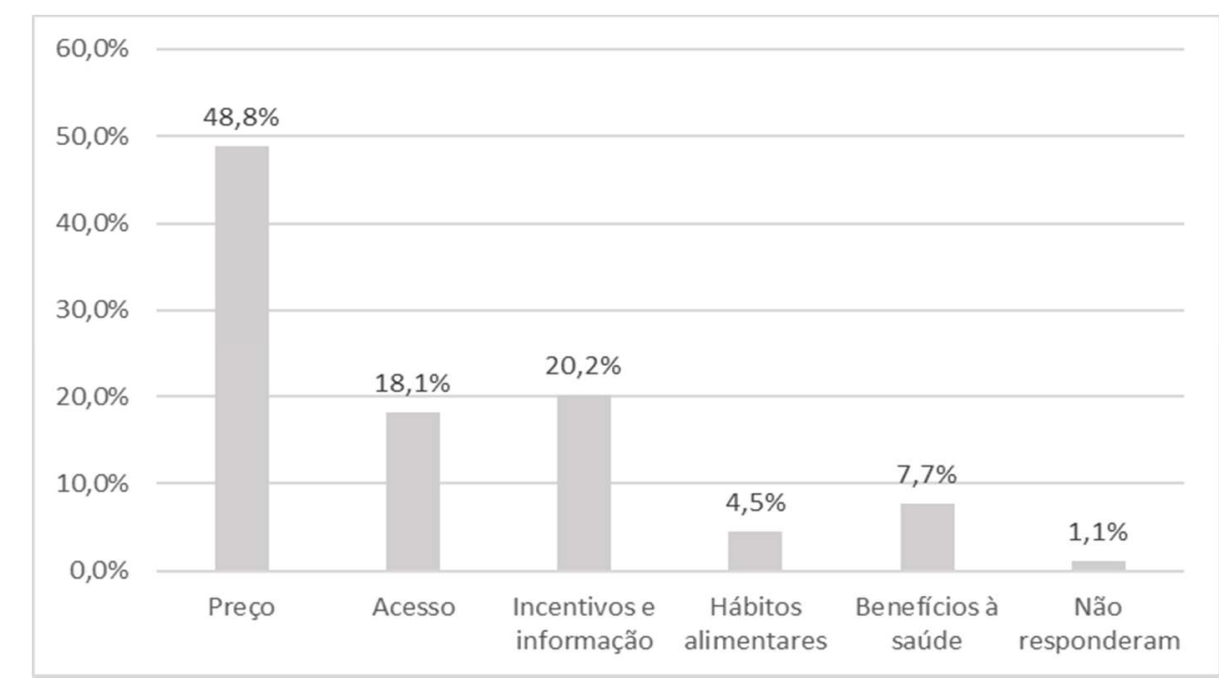

Gráfico 5 - Proporção (\%) dos motivos que fariam a população consumir mais peixes de acordo com a opinião dos entrevistados, Seara, SC, 2015.

Fonte: dados compilados pelos autores, 2014.

\section{Conclusão}

Sobre a caracterização da pesquisa, identificou-se que a população estudada era composta pela sua maioria por indivíduos do gênero feminino, com renda de um a três salários mínimos e com ensino médio completo. 
O consumo de pescados teve uma prevalência alta, no entanto se encontrava abaixo do recomendado, quando analisado o per capita anual e em comparação ao recomendado pela FAO.

O preço do pescado, pareceu ter um papel relevante na decisão de escolha, compra e consumo de peixe, pelos entrevistados, visto que dentre os motivos expostos para se elevar o consumo, estava relacionado a oferta de preços mais baixos, assim como, maior divulgação, o acesso, visto que não é uma região litorânea, e também a maior exposição de dados sobre seus benefícios relacionados à saúde.

Sendo assim, sugere-se que outros estudos preencham as lacunas da então pesquisa relacionadas ao conhecimento da população acerca dos benefícios do consumo de peixe, bem como projetos que formulem ações que auxiliem na divulgação e incentivo ao consumo de peixes, em outras regiões do país, a fim de demonstrar outras realidades, pois, o país tem uma grande área geográfica, além de uma cultura alimentar heterogênea.

Há ainda, que se ressaltar a importância do consumo deste alimento à saúde humana, evidenciada por vários estudos na literatura, mas muito se falta em relação a ações políticas e gestores; em educação nutricional a população em geral sobre os benéficos do consumo do peixe, e principalmente aos aspectos relacionados à disponibilidade de preços mais acessíveis a população brasileira.

\section{Referências}

BARBOSA, J.A.et al. Características comportamentais do consumidor de peixe no mercado de Belém. Boletim Técnico-científico do CEPNOR, Belém, v. 7, n. 1, p. 115-133, 2007.

BRASIL. Ministério da Saúde. Resolução do Conselho Nacional de Saúde n ${ }^{\circ}$ CNS no 466, de 12 de dezembro de 2012. Aprova diretriz e normas regulamentadoras de pesquisas envolvendo seres humanos. 2012. Disponível em:

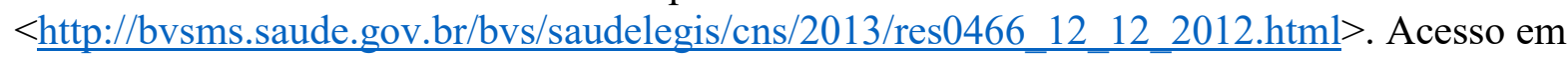
16 abril 2019.

BRASIL, Ministério da Pesca e Aquicultura, 2012. Boletim estatístico da pesca e aquicultura 2010 - Brasil. Disponível em http://www.mpa.gov.br Acesso em: 26 ago 2015.

BRASIL. Ministério da Agricultura, Pecuária e Abastecimento. Decreto no 30.691, de 29 de março de 1952. Aprova o novo Regulamento da Inspeção Industrial e Sanitária de Produtos de Origem Animal. Diário Oficial da União, Brasília, 7 jul. 1952. Seção 1, p. 10785. Disponível em:

http://extranet.agricultura.gov.br/sislegisconsulta/servlet/VisualizarAnexo?id=14013. Acesso em: 16/10/2013. 
BRASIL. Guia alimentar para a população brasileira: promovendo a alimentação saudável. Brasília: Ministério da Saúde, Secretaria de Atenção à Saúde, 2008. 210 p.

BURGER, J.. Fishing, fish consumption, and awareness about warnings in a university community in central New Jersey in 2007, and comparisons with 2004. Environmental Research, [s.1.], v. 108, n. 1, p. 107-116, 2008. Elsevier BV.

http://dx.doi.org/10.1016/j.envres.2008.05.003.

FAO, Food and Agriculture Organization of the United Nations. Statistics and Information Service of the Fisheries and Aquaculture Department. FAO yearbook. Fishery and Aquaculture Statistics. Rome, 2010.

FAO. Aspectos econômicos do mercado brasileiro de pescado. 2011. Ana Luíza de Souza Soares. Disponível em: ftp://ftp.sp.gov.br/ftppesca/3simcope/3simcope_palestra3.pdf

Food and Agriculture Organization. The State of World Fisheries and Aquaculture. Fisheries and Aquaculture Department. Rome: FAO; 2009. Acessado em: 11 ago 2014.

Instituto Brasileiro de Geografia e Estatística - IBGE. Censo Demográfico de Seara, Santa Catarina. 2010 disponível em:< https://cidades.ibge.gov.br/brasil/sc/seara/panorama>.

MACEDO, D. B. et al. Processo decisório de compra e caracterização do consumidor de peixes na cidade de Palmas - TO. Jornada de Iniciação Científica e Extensão do Instituto Federal do Tocantins, 2014, Dianópolis. V JICE, 2014.

MINOZZO, M. G. I; HARACEMIV, S. M. C. I. I; WASZCZYNSKYJ, N. Perfil dos consumidores de pescado nas cidades de São Paulo (SP), Toledo (PR) e Curitiba (PR) no Brasil. Revista da Spcna: Alimentação Humana, Curitiba, v. 14, n. 3, p.133-140, 2008.

NOMURA, Ichiro. O futuro da pesca e da aquicultura marinha no mundo. Ciência e cultura. Vol. 63. No. 3. São Paulo, 2010.

PEREIRA, M. P.; TELLES, E. O.; DIAS, R. A.; BALIAN, S. C. Descrição do sistema agroindustrial brasileiro de pescado. Informações Econômicas, v. 40, n. 3, p. 54-61, 2010.

PORTAL BRASIL. Cresce consumo de pescado entre brasileiros. 2013. Disponível em: http://www.brasil.gov.br/economia-e-emprego/2013/08/cresce-consumo-de-pescadoentrebrasileiros. Acesso em: 11 ago. 2014.

PRADO, S.P.T.; CAPUANO, D.M. Relato de nematóides da família Anisakidae em bacalhau comercializado em Ribeirão Preto, São Paulo. Revista da Sociedade Brasileira de Medicina Tropical, v.39, n 6, p. 580-581, 2006.

SARTORI, A. G. DE O.; AMANCIO, R. D. Pescado: importância nutricional e consumo no Brasil. Segurança Alimentar e Nutricional, v. 19, n. 2, p. 83-93, 2012.

VASCONCELLOS, Juliana Parreira. Determinantes do consumo de pescado pela população que frequenta feiras livres no município de Santo André, SP. 2010. 104 p. Dissertação (Mestrado em Epidemiologia Experimental Aplicada à Zoonoses) Universidade de São Paulo, São Paulo, 2010. 


\title{
DOES THE POPULATION CONSUME THE AMOUNT OF FISH RECOMMENDED BY FAO?
}

\begin{abstract}
In the last decades aquaculture is receiving great attention because of its health-related benefits. The objective of this work was to verify the socioeconomic profile and identify the fish consumption of individuals residing in the municipality of Seara, located in the west of Santa Catarina state, as well as the quantity they consume and the reasons that would make them consume more fish. The research has a quali-quantitative, descriptive, and transversal nature. 376 individuals were interviewed, with a questionnaire consisting of 30 objective and subjective questions. This research project was submitted to and approved by the Ethics and Research Committee of the Universidade Federal da Fronteira Sul (UFFS). The results showed that as the level of education and income increases, so does the amount of fish consumed by the interviewees. It was noticed that a large part of the population consumes fish, however the amount consumed is below the recommended. Among the reasons that would make the population increase their fish intake are more accessible prices and incentives.
\end{abstract}

Keywords: food consumption; fish; nutritional recommendation; aquaculture.

\section{¿CONSUME LA POBLACIÓN LA CANTIDAD DE PECES RECOMENDADA POR LA FAO?}

Resumen: En las últimas décadas, la acuicultura ha recibido gran atención debido a sus beneficios relacionados con la salud. El objetivo de este trabajo fue verificar el perfil socioeconómico e identificar el consumo de pescado de las personas que residen en el municipio de Seara, ubicado en el oeste de Santa Catarina, así como la cantidad consumida y las razones que los harían consumir más pescado. La investigación fue de naturaleza cualitativa, descriptiva y transversal. Se entrevistó a 376 personas, donde se aplicó un cuestionario compuesto por 30 preguntas objetivas y subjetivas. Este proyecto de investigación fue presentado y aprobado por el Comité de Ética e Investigación de la Universidade Federal da Fronteira Sul (UFFS). Los resultados mostraron que a medida que aumenta el nivel de educación e ingresos, también aumenta la cantidad de pescado consumido por los entrevistados. Se notó que una gran parte de la población consume pescado, sin embargo, la cantidad consumida está por debajo de lo recomendado. Entre las razones que harían que la población aumentara su consumo de pescado están los precios e incentivos más accesibles.

Palabras clave: consumo de alimentos; pez; recomendación nutricional; acuicultura 